\title{
The Field and Energy Distributions of the Fundamental Mode in the Solid-Core Photonic Crystal Fibers for Different Geometric Parameters
}

\author{
Halime Demir Inci and Sedat Ozsoy \\ Department of Physics, Faculty of Science and Arts, Erciyes University, 38039 Kayseri, Turkey \\ Correspondence should be addressed to Sedat Ozsoy; ozsoys@erciyes.edu.tr
}

Received 16 June 2013; Accepted 12 July 2013

Academic Editors: Y. S. Kivshar and S. Ponomarenko

Copyright (C) 2013 H. Demir Inci and S. Ozsoy. This is an open access article distributed under the Creative Commons Attribution License, which permits unrestricted use, distribution, and reproduction in any medium, provided the original work is properly cited.

For a solid core photonic crystal fiber with the triangular lattice, the field and energy distributions of fundamental mode are considered at $1.55 \mu \mathrm{m}$ wavelength. The silica core is constituted by removing the 7 air holes. The cladding consists of the twodimensional silica-air photonic crystal with the 4 rings of air holes. The field and energy distributions were investigated for three different values of $d / \Lambda$. Here, $d$ and $\Lambda$ represent the diameter of air holes and the pitch length, respectively. The simulations show that, for the fixed $\Lambda$, the increase in $d / \Lambda$ ratio does not cause the considerable changes in the field and energy intensity distributions, but for the fixed $d$, the increase in this ratio affects the intensity distributions reasonably.

\section{Introduction}

In recent years, the photonic crystal fibers (PCFs) have been of significant interest due to their unique structures and new properties [1-6]. Generally, photonic crystal fibers consist of an arrangement of air holes in the cladding extending the whole length of the fiber. Photonic crystal fibers are categorized into two groups according to light guiding mechanism. One is the index guiding photonic crystal fiber, and the other is the photonic band gap PCF. In the index guiding PCFs, the core region is solid and the light is confined in the central core as in the conventional fibers. The photonic crystal fiber consists of the pure silica fiber with an array of the air holes along the length of the fiber. The core is constituted by removing the central hole from the structure. The higher effective refractive index of the surrounding holes forms cladding in which leading the index guiding mechanism analogous to total internal reflection. Consequently, the light guiding can be explained by the total internal reflection which is also the way light is guided in step index fibers. If around the central air hole there is the two-dimensional photonic crystal cladding which consists of a periodic array of the silica and air, such fibers are called the photonic band gap photonic crystal fibers. The central hole in these fibers acts as the core, and in this place the light is guided by the photonic band gap effect. The frequencies within the band gap of the structure will expose to the multiple Bragg reflection that leads to the destructive interference of the light trying to propagate away from the air core. The function of the air core is to provide a defect at the periodic structure that the propagation of the frequencies within the photonic band gap is really allowed. Therefore, it is not needed for the core indexes of these fibers to be bigger than the effective index of cladding.

PCFs have been shown to possess many important properties as the single mode operation over wide range of wavelength, the highly tunable dispersion, the propagation of high power densities without exciting unwanted nonlinear effects, and the high birefringence. These properties have the practical importance in the design of sophisticated broadband optical telecommunication networks [7] and active sensor systems [8].

In this work, the software based on the finite element method (FEM) is used for the field and energy distribution simulations of the fundamental mode at the solid core photonic crystal fiber structures with the different geometrical parameters. 


\section{Theoretical Model}

The large index contrast and complex structure in the photonic crystal fibers make them difficult to be treated mathematically. The standard optical fiber analysis does not help, and moreover in the majority of photonic crystal fibers, it is practically impossible to perform modal analysis analytically; therefore the Maxwell equations must be solved numerically. The main idea consists of transforming this complicated problem, which can be described by the curl-curl equation [9]:

$$
\nabla \times\left(\mu_{r}^{-1} \nabla \times \vec{E}\right)-k_{0}^{2} \varepsilon_{r} \vec{E}=0 .
$$

This is an eigenvalue equation, the eigenvalues are $\beta / k_{0}$ the effective indexes, and the eigenvectors are the electric field components $\left(E_{x}, E_{y}, E_{z}\right)$. In (1), $\vec{E}$ is the electric field, $k_{0}$ is the wavenumber in vacuum, and $\varepsilon_{r}$ and $\mu_{r}$ are the dielectric permittivity and magnetic permeability tensors, respectively.

Here in the analysis of the properties of PCFs, the FEM is used. The FEM allows the photonic crystal fiber crosssection in the $x-y$ plane to be divided into a patchwork of triangular elements in which they can possess different sizes, shapes, and refractive indexes. In this way, any geometry including the PCF air hole and the medium characteristics can be accurately described. In particular, the FEM is suited for studying fibers with the nonperiodic arrangements of the air holes. However, it provides a full vectorial analysis that is necessary to model the PCFs with the large air holes and high index variations and to accurately predict the properties of these fibers [10].

The FEM is basically divided in to four steps [11]. The domain discretization is the first and perhaps the most important step in any finite element method. The first step consists of dividing the solution domain $\Omega$ into the subdomains of finite number in which form a patchwork of fundamental elements that can possess different sizes, shapes, and physical properties. The second step is choosing the interpolation functions that provide an approximation of the unknown solution within each element. In this specific case, the solution domain is the transverse cross-section of optical waveguide that is divided in triangular finite elements, and the nodal approach is followed using secondorder polynomials as interpolating functions in each finite element (each triangular is characterized by 6 nodes, and the unknowns are the magnetic field components). In the next step, the curl-curl equation is transformed into a generalized eigenvalue problem by applying the variational formulation as follows:

$$
[A]\{\Phi\}-\lambda[B]\{\Phi\}=\{0\},
$$

where the eigenvalue $\lambda$ is the mode effective refractive index $\left(n_{\text {eff }}\right)$ and the eigenvector $\Phi$ is the full vectorial electric distribution $\left(E_{x}, E_{y}, E_{z}\right)$ in the nodal points.

Finally, the fourth and final step in the finite element analysis is to solve the eigenvalue system. The matrices $[A]$ and $[B]$ are sparse and symmetric; therefore, the computational time can be effectively minimized by using the sparse matrix solver.

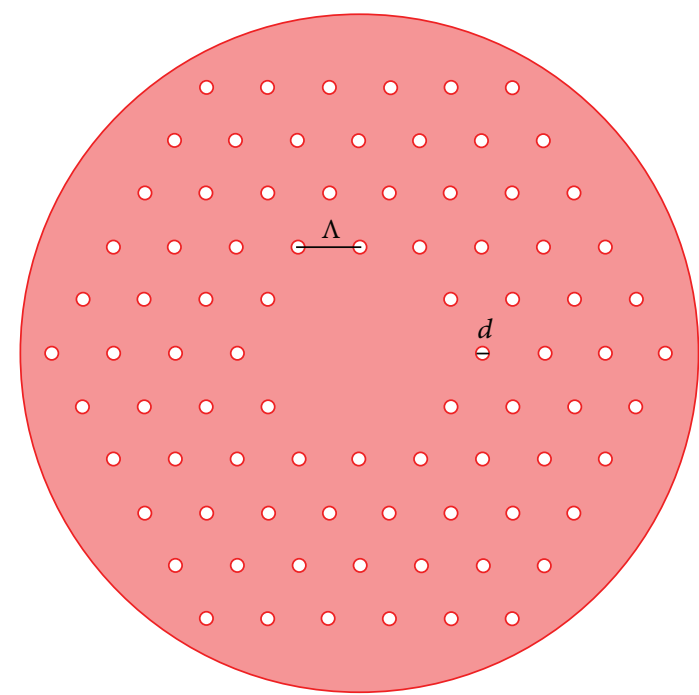

FIgure 1: The cross-section of the solid core PCF considered. $\Lambda$ is the pitch length, and $d$ is the diameter of air holes.

TABLE 1: The effective refractive indexes of the fundamental mode for different $d / \Lambda$ ratios.

\begin{tabular}{lccc}
\hline & $d / \Lambda$ & $\lambda / \Lambda$ & $n_{\text {eff }}\left(\lambda=1.55 \mu \mathrm{m}, n_{\text {silica }}=1.44409\right)$ \\
\hline & 0.08 & & $1.442911-2.534351 e-8 i$ \\
& 0.09 & & $1.442822-1.378536 e-8 i$ \\
& 0.10 & & $1.442747-5.387208 e-9 i$ \\
& 0.20 & & $1.442334-4.984438 e-12 i$ \\
Fixed pitch & 0.30 & & $1.442108-2.353948 e-14 i$ \\
$(\Lambda=4.2 \mu \mathrm{m})$ & 0.40 & 0.37 & $1.441914-8.43704 e-17 i$ \\
& 0.50 & & $1.441721-2.22923 e-19 i$ \\
& 0.60 & & $1.44152-3.398141 e-19 i$ \\
& 0.70 & & $1.441303-5.284044 e-19 i$ \\
& 0.80 & & $1.441068-4.492512 e-20 i$ \\
& 0.90 & & $1.440809-3.595418 e-19 i$ \\
\hline & 0.08 & 0.15 & $1.443843-7.982422 e-11 i$ \\
& 0.09 & 0.17 & $1.443773-9.149398 e-11 i$ \\
& 0.10 & 0.19 & $1.443693-4.61459 e-11 i$ \\
& 0.20 & 0.37 & $1.442334-4.984438 e-12 i$ \\
$(d=0.84 \mu \mathrm{m})$ & 0.30 & 0.56 & $1.439848-1.198296 e-12 i$ \\
& 0.40 & 0.71 & $1.436078-1.976832 e-13 i$ \\
0.50 & 0.91 & $1.43085-8.180793 e-14 i$ \\
& 0.60 & 1.11 & $1.423967-3.413292 e-14 i$ \\
& 0.70 & 1.25 & $1.41519-2.279162 e-14 i$ \\
& 0.80 & 1.48 & $1.404232-2.485407 e-14 i$ \\
0.90 & 1.67 & $1.390737-3.793315 e-14 i$ \\
\hline & & &
\end{tabular}

\section{Simulation Results}

For the simulations, the photonic crystal fiber with crosssection shown in Figure 1 is investigated at $1.55 \mu \mathrm{m}$ wavelength. Here $\Lambda$ is the pitch length, and $d$ is the diameter of air holes. The fiber core is silica, and it is formed by removing 


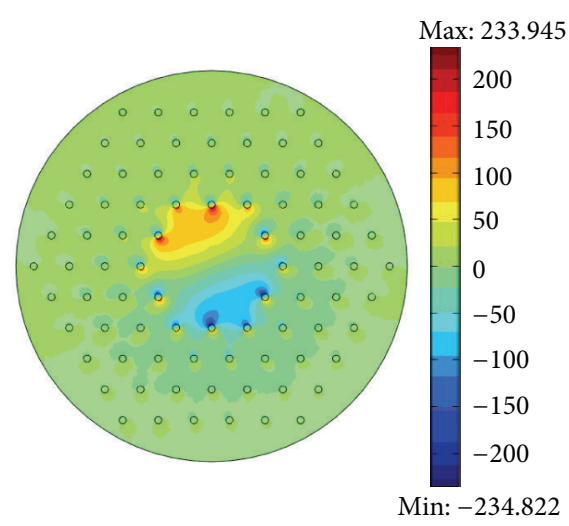

(a)

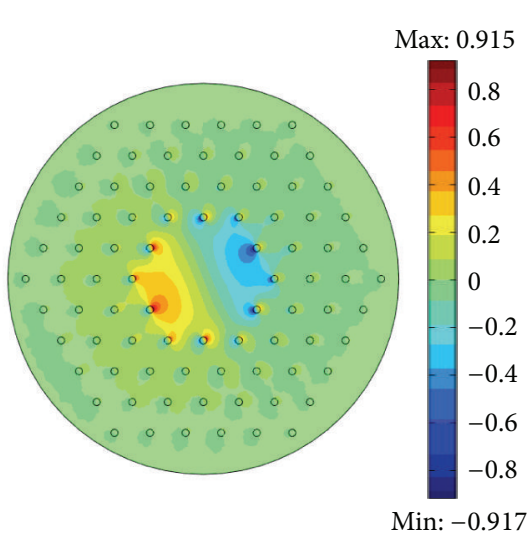

(b)

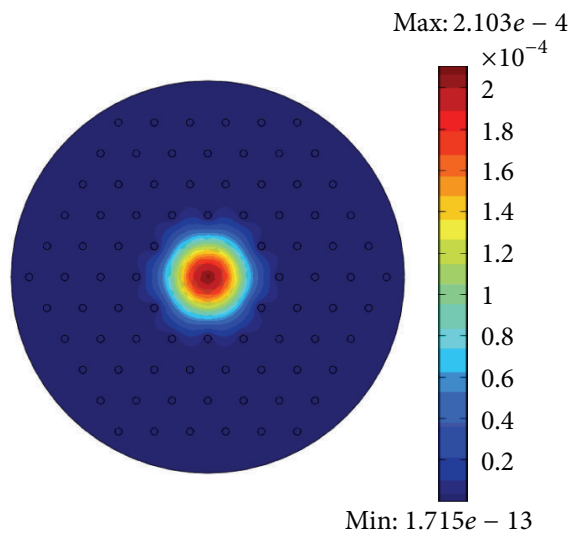

(c)

Figure 2: For $\Lambda=4.2 \mu \mathrm{m}$ and $d / \Lambda=0.2$, (a) the distribution of $z$-component of the electric field, (b) the distribution of $z$-component of the magnetic field, and (c) the total energy distribution.

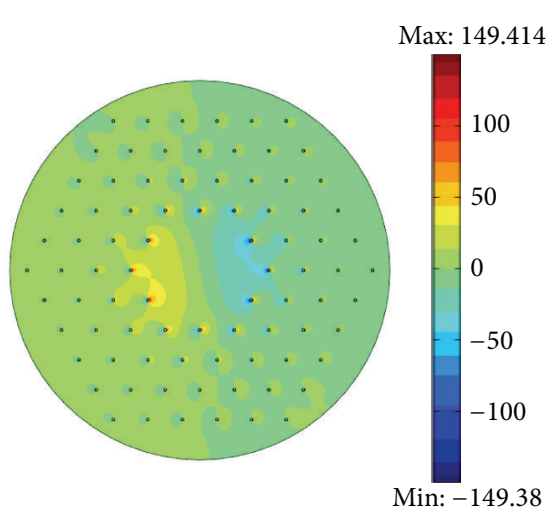

(a)

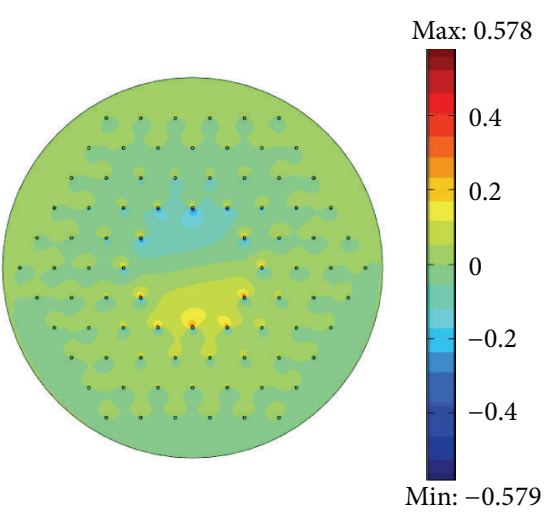

(b)

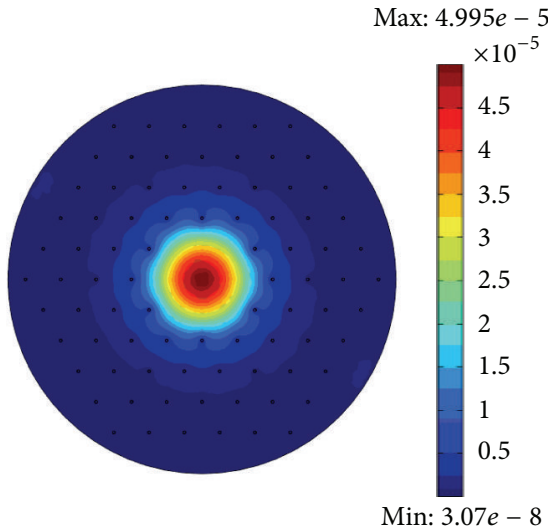

(c)

FIGURE 3: For $\Lambda=4.2 \mu \mathrm{m}$ and $d / \Lambda=0.08$, (a) the distribution of $z$-component of the electric field, (b) the distribution of $z$-component of the magnetic field, and (c) the total energy distribution.

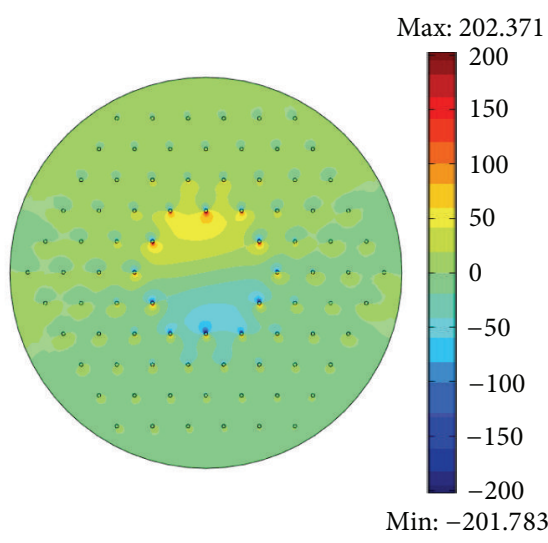

(a)

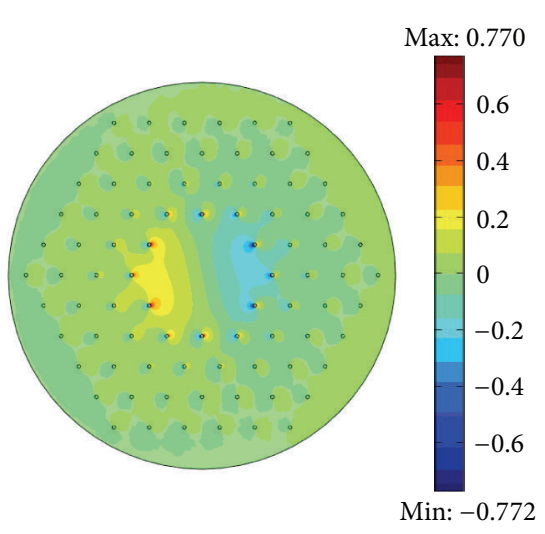

(b)

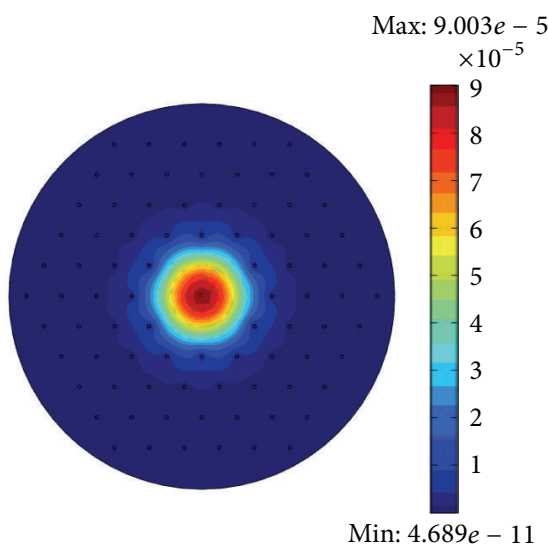

(c)

FIGURE 4: For $\Lambda=4.2 \mu \mathrm{m}$ and $d / \Lambda=0.1$, (a) the distribution of $z$-component of the electric field, (b) the distribution of $z$-component of the magnetic field, and (c) the total energy distribution. 


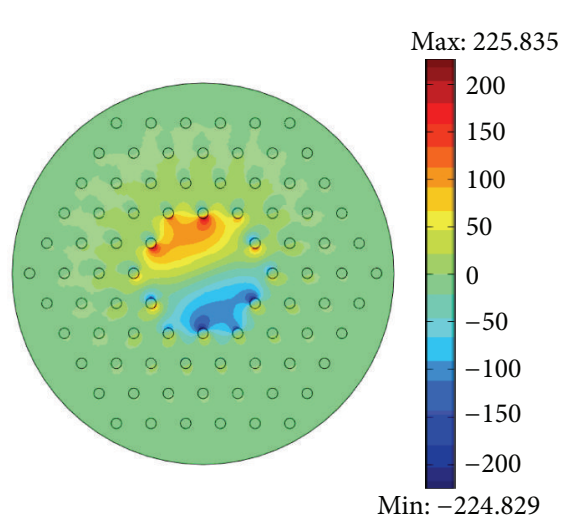

(a)

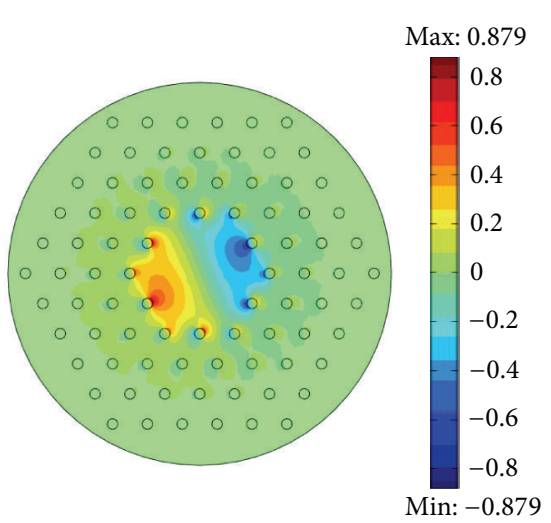

(b)

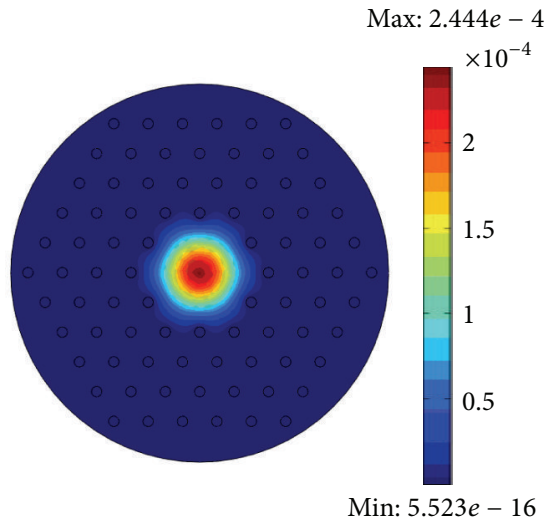

(c)

FIGURE 5: For $\Lambda=4.2 \mu \mathrm{m}$ and $d / \Lambda=0.3$, (a) the distribution of $z$-component of the electric field, (b) the distribution of $z$-component of the magnetic field, and (c) the total energy distribution.

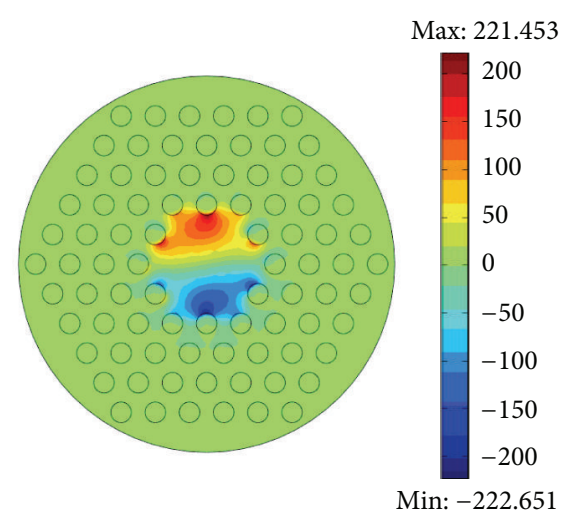

(a)

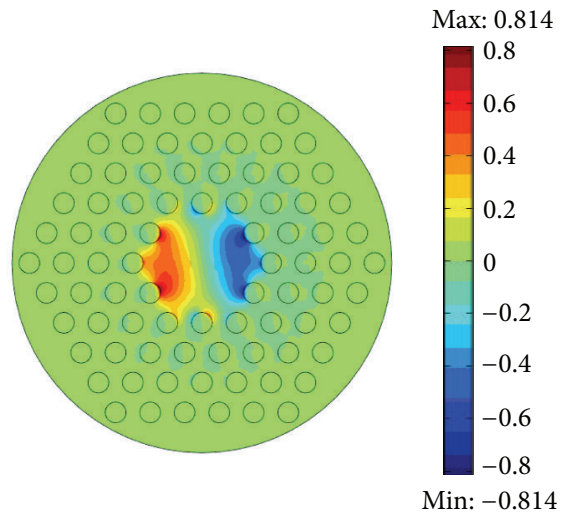

(b)

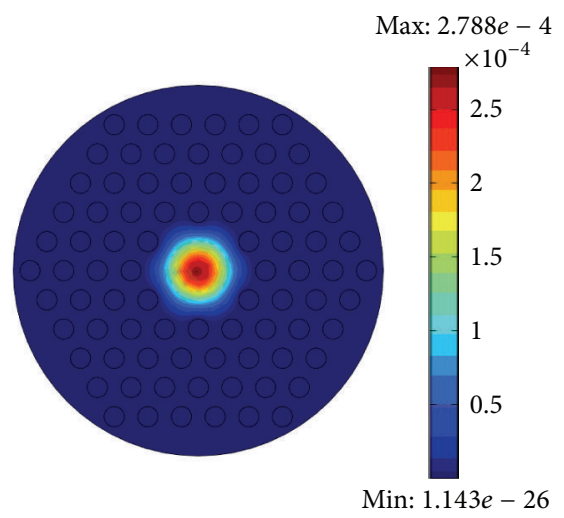

(c)

FIGURE 6: For $\Lambda=4.2 \mu \mathrm{m}$ and $d / \Lambda=0.6$, (a) the distribution of $z$-component of the electric field, (b) the distribution of $z$-component of the magnetic field, and (c) the total energy distribution.

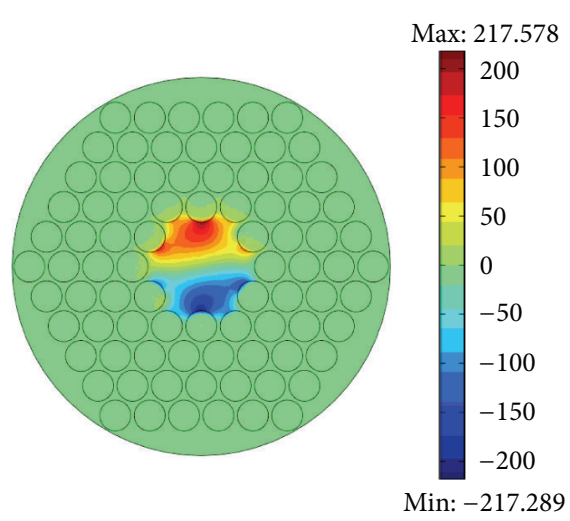

(a)

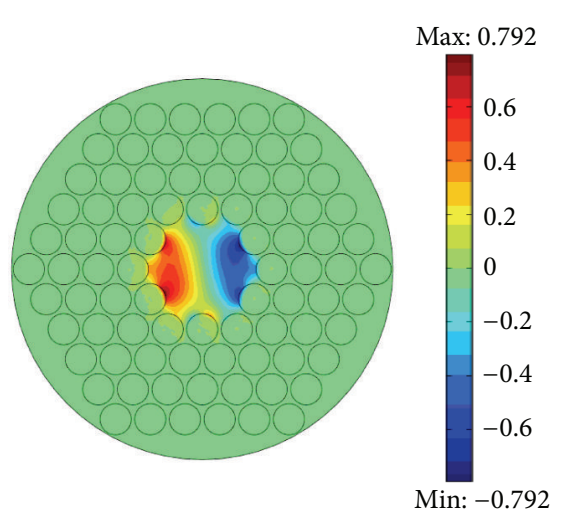

(b)

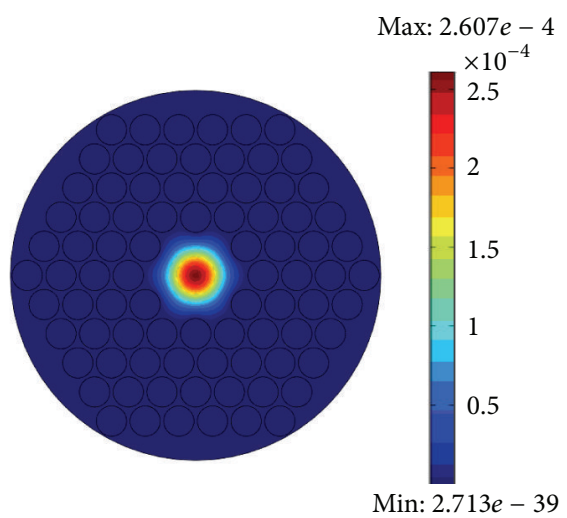

(c)

FIGURE 7: For $\Lambda=4.2 \mu \mathrm{m}$ and $d / \Lambda=0.9$, (a) the distribution of $z$-component of the electric field, (b) the distribution of $z$-component of the magnetic field, and (c) the total energy distribution. 


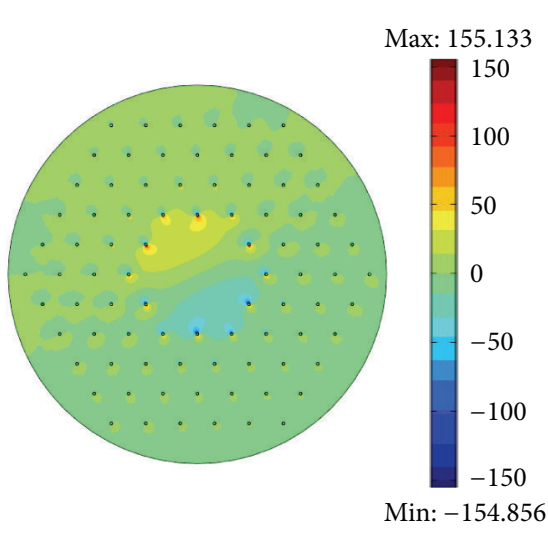

(a)

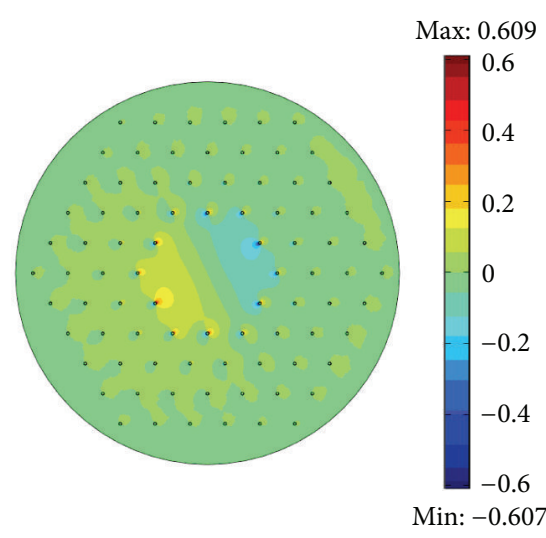

(b)

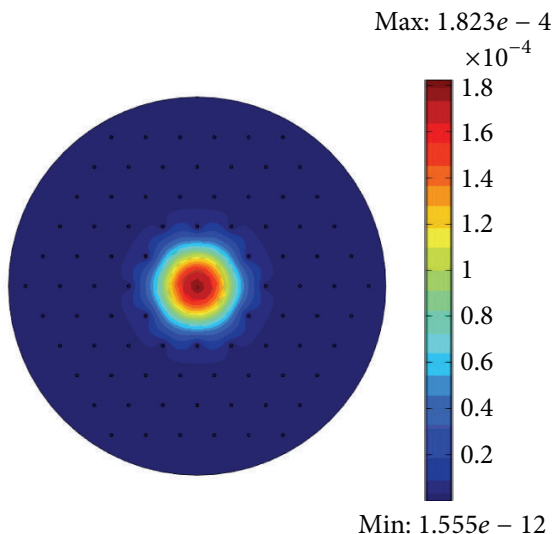

(c)

Figure 8: For $d=0.84$ and $d / \Lambda=0.08 \mu \mathrm{m}$, (a) the distribution of $z$-component of the electric field, (b) the distribution of $z$-component of the magnetic field, and (c) the total energy distribution.

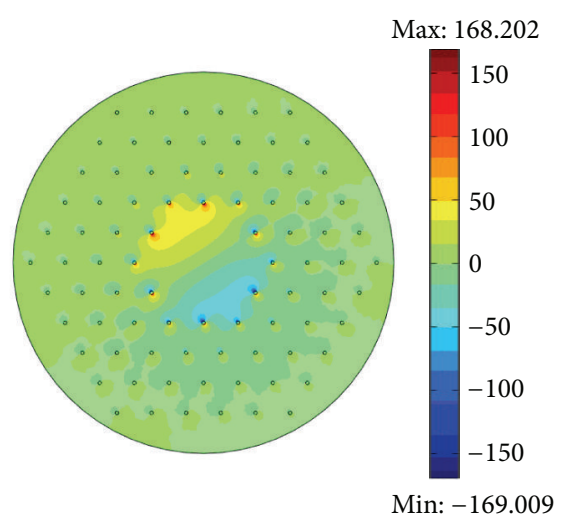

(a)

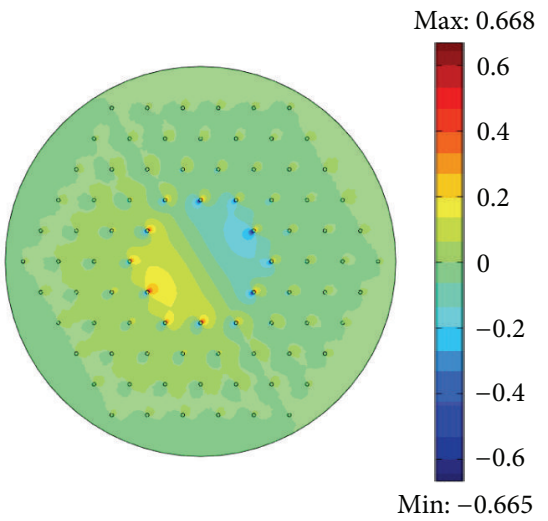

(b)

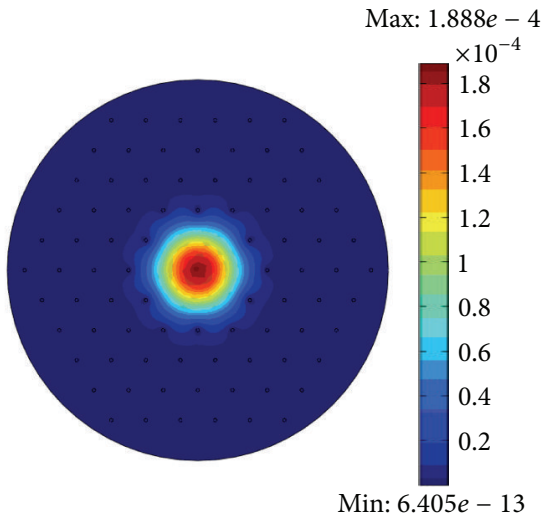

(c)

Figure 9: For $d=0.84$ and $d / \Lambda=0.1 \mu \mathrm{m}$, (a) the distribution of $z$-component of the electric field, (b) the distribution of $z$-component of the magnetic field, and (c) the total energy distribution.

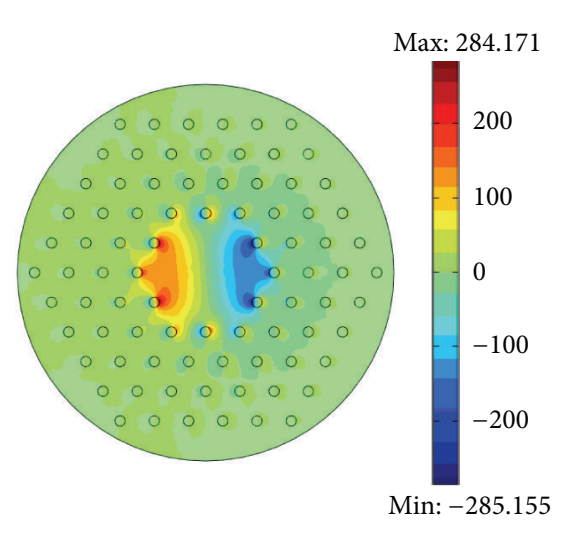

(a)

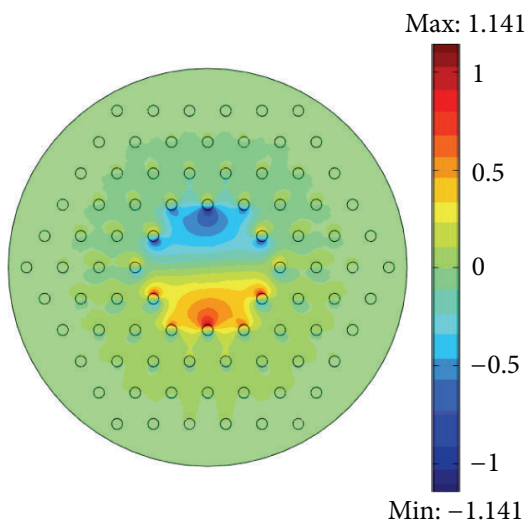

(b)

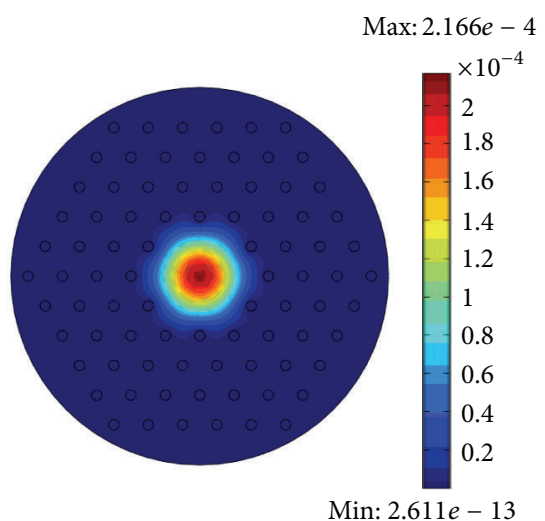

(c)

Figure 10: For $d=0.84 \mu \mathrm{m}$ and $d / \Lambda=0.3 \mu \mathrm{m}$, (a) the distribution of $z$-component of the electric field, (b) the distribution of $z$-component of the magnetic field, and (c) the total energy distribution. 


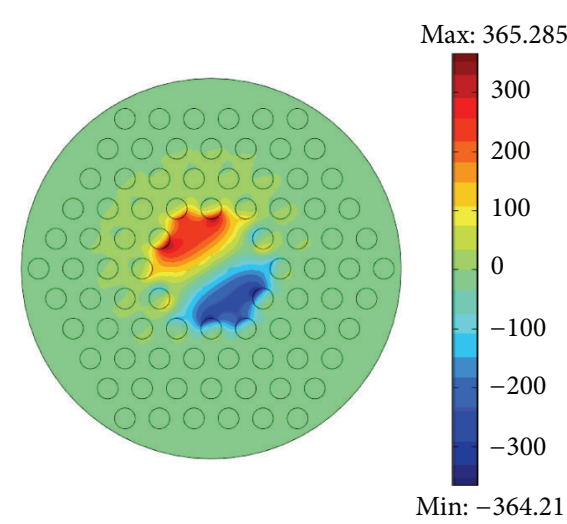

(a)

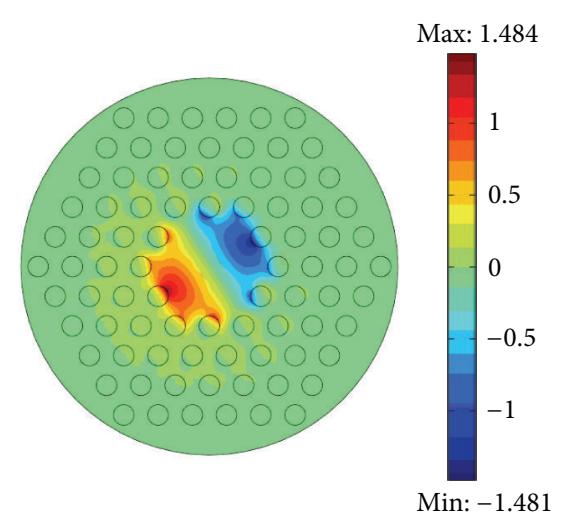

(b)

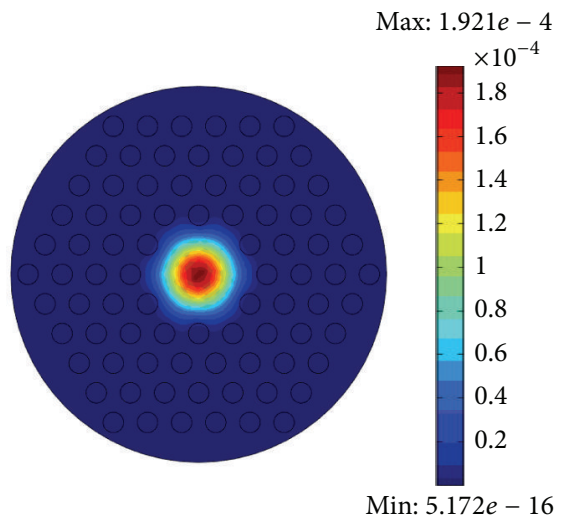

(c)

FIGURE 11: For $d=0.84 \mu \mathrm{m}$ and $d / \Lambda=0.6 \mu \mathrm{m}$, (a) the distribution of $z$-component of the electric field, (b) the distribution of $z$-component of the magnetic field, and (c) the total energy distribution.

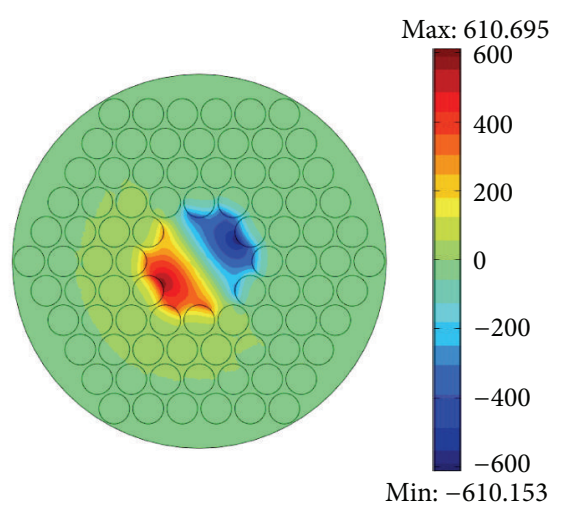

(a)

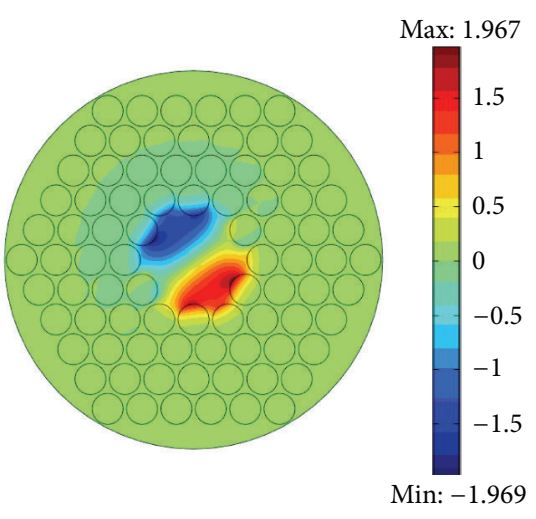

(b)

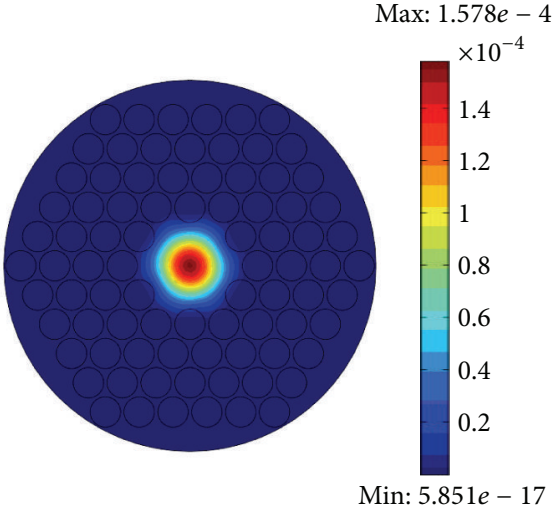

(c)

Figure 12: For $d=0.84 \mu \mathrm{m}$ and $d / \Lambda=0.9 \mu \mathrm{m}$, (a) the distribution of $z$-component of the electric field, (b) the distribution of $z$-component of the magnetic field, and (c) the total energy distribution.

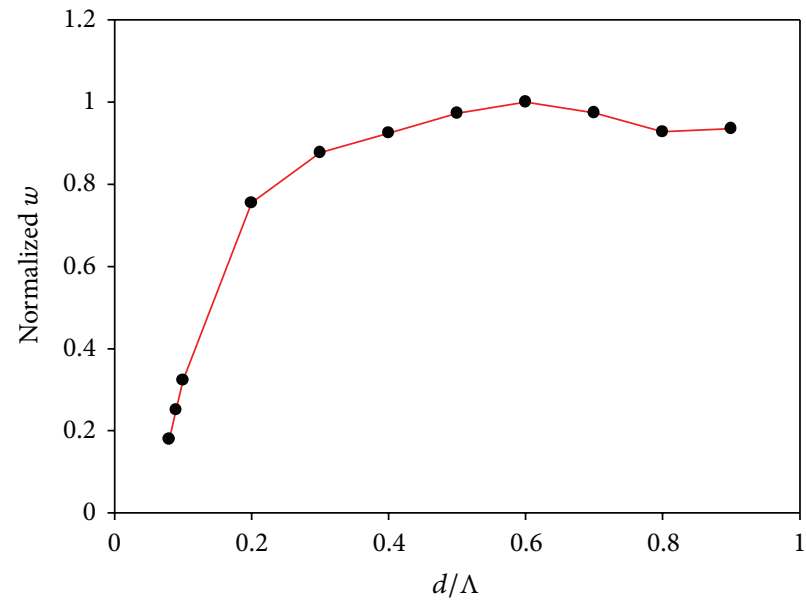

(a)

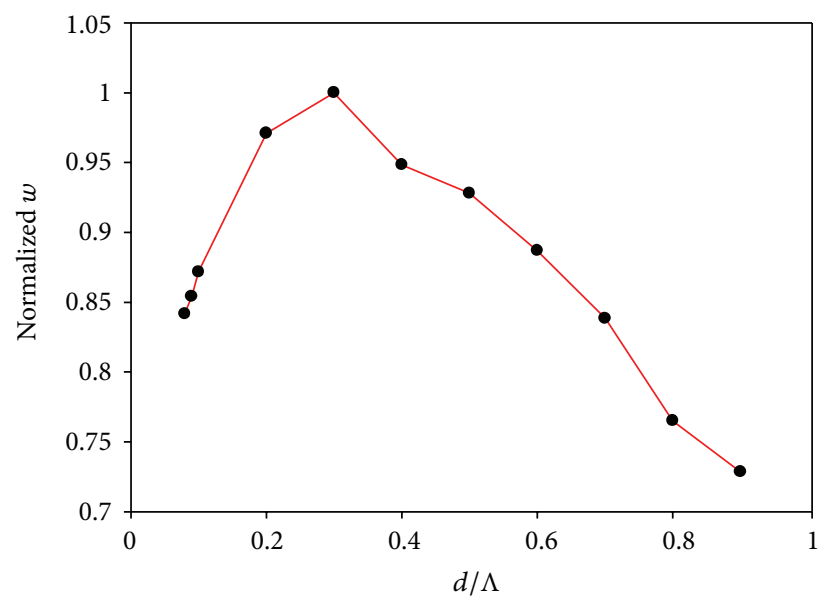

(b)

FIGURE 13: The normalized total energy of the fundamental mode at the central of the core for different $d / \Lambda$ ratios for (a) fixed pitch $\Lambda=4.2 \mu \mathrm{m}$ and (b) fixed diameter $d=0.84 \mu \mathrm{m}$. 


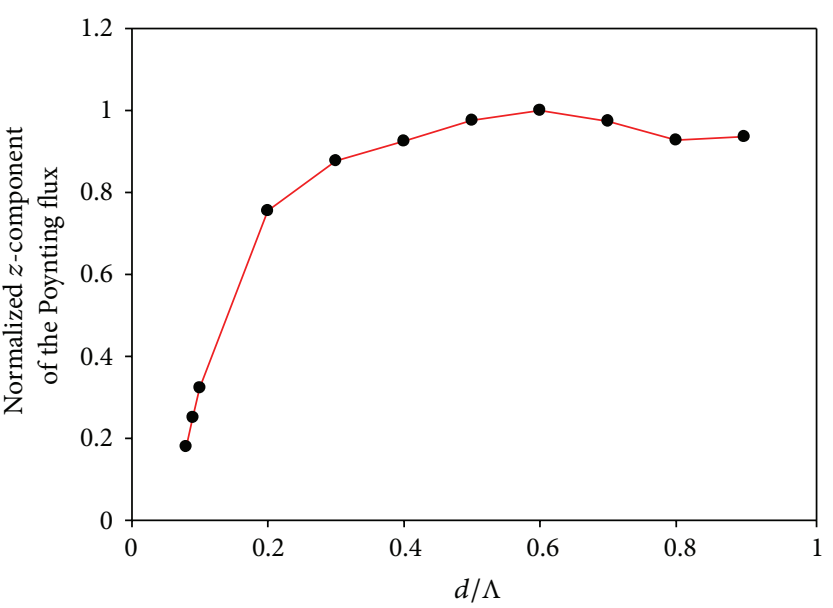

(a)

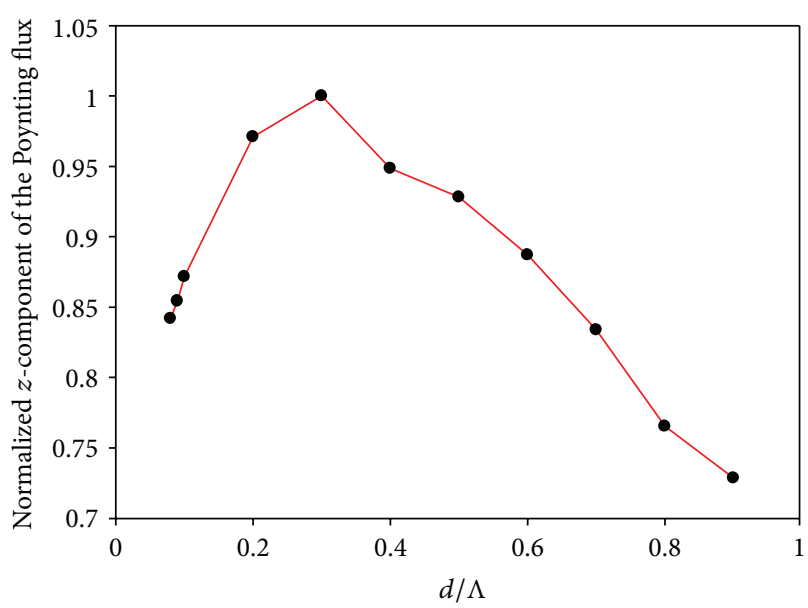

(b)

Figure 14: The normalized $z$-component of the Poynting flux of the fundamental mode at the central of the core for different $d / \Lambda$ ratios for (a) fixed pitch $\Lambda=4.2 \mu \mathrm{m}$ and (b) fixed diameter $d=0.84 \mu \mathrm{m}$.

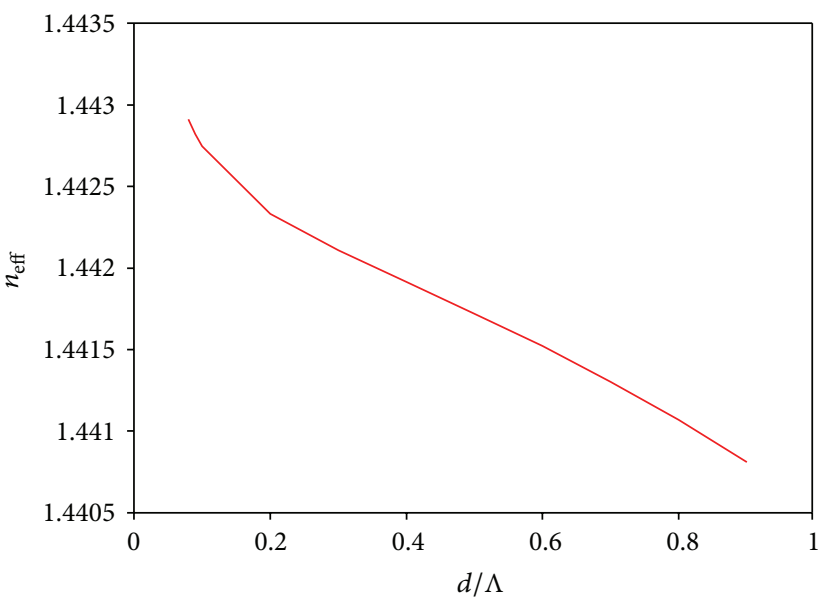

(a)

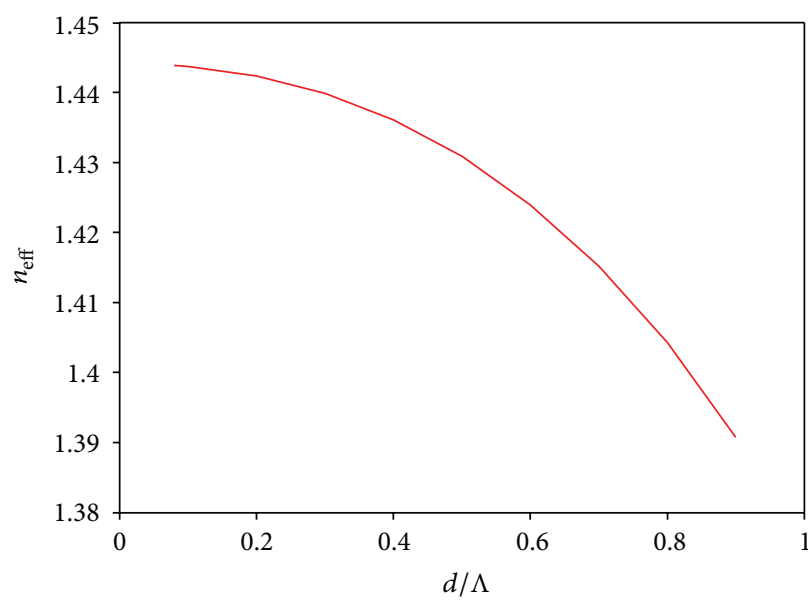

(b)

Figure 15: The effective refractive indexes of the fundamental mode for different $d / \Lambda$ ratios for (a) fixed pitch $\Lambda=4.2 \mu \mathrm{m}$ and (b) fixed diameter $d=0.84 \mu \mathrm{m}$.

7 air holes from the structure. The cladding is the twodimensional photonic crystal with 4 rings of the triangular lattice air holes in the silica matrix.

Firstly, for the fixed pitch length $\Lambda=4.2 \mu \mathrm{m}$, the field and energy distributions are investigated by varying the diameters of air holes for the $d / \Lambda$ values of $0.1,0.2,0.3,0.4,0.5,0.6,0.7$, 0.8 , and 0.9. Later, a similar investigation is executed for the fixed diameter of air-hole $d=0.84 \mu \mathrm{m}$, by varying the pitch length.

For $\Lambda=4.2 \mu \mathrm{m}$ and the cross-section with the radius of $23.1 \mu \mathrm{m}$, the electric and magnetic fields and the energy distributions of the structure for $d / \Lambda=0.2,0.08,0.1,0.3,0.6$, and 0.9 are shown in Figures 2, 3, 4, 5, 6, and 7, respectively.

For a given pitch length, $\Lambda=4.2 \mu \mathrm{m}$, the electric and magnetic field distributions are more extended into the cladding region (i.e., guiding is weakened) when the $d / \Lambda$ ratio reduces (Figures 2-9). In other words, the light is well confined in the core when the $d / \Lambda$ ratio increases. For $d / \Lambda=$ 0.08 , the effective index of the fundamental mode becomes complex, and thus the mode becomes leaky. As a result of this, the energy intensity transported in the core region is also reduced. The reason of this behavior is that the effective cladding index approaches the core index, and hence the index difference decreases.

For a fixed hole diameter, $d=0.84 \mu \mathrm{m}$, Figures 9 and 10 show the fields and energy distributions of the structure for $d / \Lambda=0.1$ and the cross-section with the radius of $46.2 \mu \mathrm{m}$ and $d / \Lambda=0.3$ and the cross-section with the radius of $15.4 \mu \mathrm{m}$, respectively. In addition, Figures 11 and 12 show the fields and energy distributions of the structure for $d / \Lambda=0.6$ and the cross-section with the radius of $7.7 \mu \mathrm{m}$ and $d / \Lambda=0.9$ and the cross-section with the radius of $5.13 \mu \mathrm{m}$, respectively.

Figures 2, 8, and 9 show that, for a given diameter of the air holes, the confinement and the total energy confined in 
the core region increase when the $d / \Lambda$ ratio increases up to a certain $d / \Lambda$ value, for which the effective index becomes complex (see Table 1). However, when the $d / \Lambda$ ratio increases above this value, it can be seen that the confinement is lower and the energy involved in the core region decreases (see Figures 2 and 10). This behaviour of the energy can be seen more clearly from Figure 13 and attributed to the mode becoming leaky due to approaching its cutoff. In addition, $z$-component of the Poynting flux of the fundamental mode versus $d / \Lambda$ values is illustrated in Figure 14 .

For the different $d / \Lambda$ ratios, the effective index variation of the fundamental mode is given in Table 1 . The effective indexes are obtained by using cylindrical PML condition. Figure 15 shows the real part of the effective refractive index of the fundamental mode versus $d / \Lambda$ ratios for both fixed pitch and fixed diameter.

\section{Conclusion}

In this study, the effect of the diameters of air holes and the pitch length on the field and energy distributions of the fundamental mode in a solid core PCF with the triangular lattice of air holes in the silica matrix is investigated. For a given pitch length, the confinement and the energy density for the fundamental mode decrease when $d / \Lambda$ decreases. For a given diameter of the air holes, the confinement and the total energy confined in the core region increase when the $d / \Lambda$ ratio increases up to a certain $d / \Lambda$ value, for which the effective index becomes complex. However, when the $d / \Lambda$ ratio increases above this value, the confinement is lower and the energy involved in the core region decreases. This behavior of the energy can be attributed to the mode becoming leaky due to approaching its cutoff.

\section{References}

[1] J. C. Knight, T. A. Birks, P. S. J. Russell, and D. M. Atkin, "Allsilica single-mode optical fiber with photonic crystal cladding," Optics Letters, vol. 21, no. 19, pp. 1547-1549, 1996.

[2] J. C. Knight, "Photonic crystal fibres," Nature, vol. 424, no. 6950, pp. 847-851, 2003.

[3] S. Arismar Cerqueira Jr., F. Luan, C. M. B. Cordeiro, A. K. George, and J. C. Knight, "Hybrid photonic crystal fiber," Optics Express, vol. 14, no. 2, pp. 926-931, 2006.

[4] T. A. Birks, J. C. Knight, and P. S. J. Russell, "Endlessly singlemode photonic crystal fiber," Optics Letters, vol. 22, no. 13, pp. 961-963, 1997.

[5] A. Ortigosa-Blanch, J. C. Knight, W. J. Wadsworth et al., "Highly birefringent photonic crystal fibers," Optics Letters, vol. 25, no. 18, pp. 1325-1327, 2000.

[6] W. J. Wadsworth, A. Ortigosa-Blanch, J. C. Knight, T. A. Birks, T.-P. M. Man, and P. S. J. Russell, "Supercontinuum generation in photonic crystal fibers and optical fiber tapers: a novel light source," Journal of the Optical Society of America B, vol. 19, no. 9, pp. 2148-2155, 2002.

[7] M. D. Nielsen, J. R. Folkenberg, N. A. Mortensen, and A. Bjarklev, "Bandwidth comparison of photonic crystal fibers and conventional single-mode fibers," Optics Express, vol. 12, no. 3, pp. 430-435, 2004.
[8] S. O. Konorov, A. M. Zheltikov, and M. Scalora, "Photoniccrystal fiber as a multifunctional optical sensor and sample collector," Optics Express, vol. 13, no. 9, pp. 3454-3459, 2005.

[9] K. Saitoh and M. Koshiba, "Single-polarization single-mode photonic crystal fibers," IEEE Photonics Technology Letters, vol. 15, no. 10, pp. 1384-1386, 2003.

[10] T. M. Monro, D. J. Richardson, N. G. R. Broderick, and P. J. Bennett, "Modeling large air fraction holey optical fibers," Journal of Lightwave Technology, vol. 18, no. 1, pp. 50-56, 2000.

[11] J. Jin, The Finite Element Method in Electromagnetics, Wiley, New York, NY, USA, 2nd edition, 2002. 

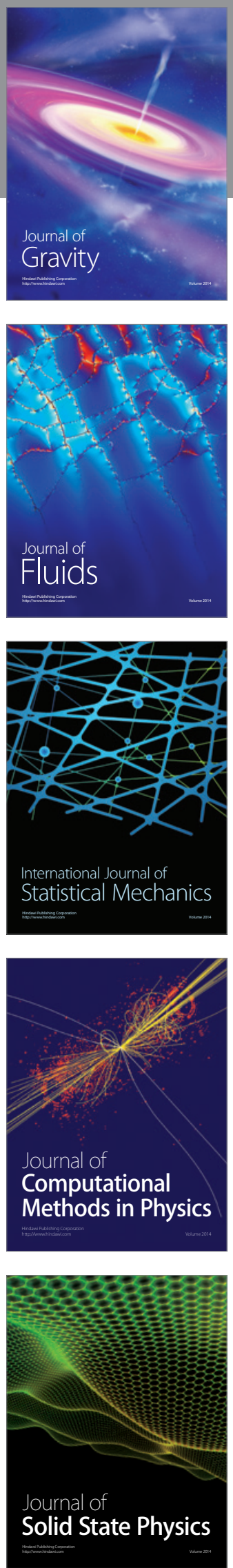

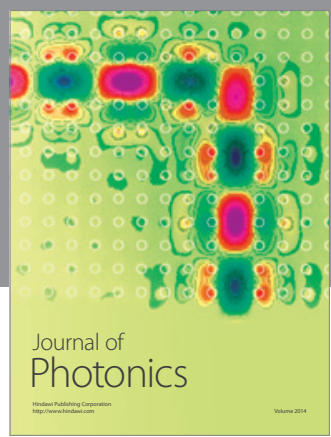

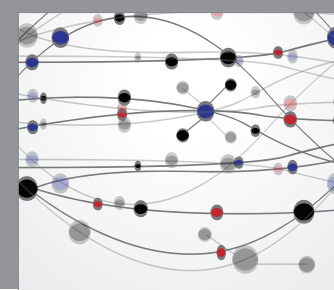

The Scientific World Journal

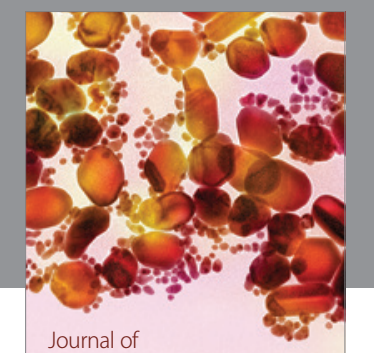

Soft Matter
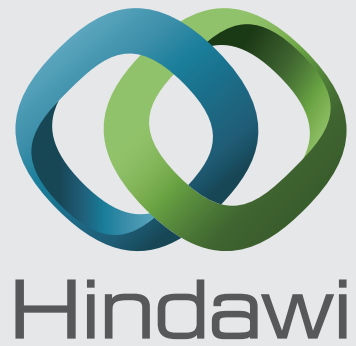

Submit your manuscripts at

http://www.hindawi.com
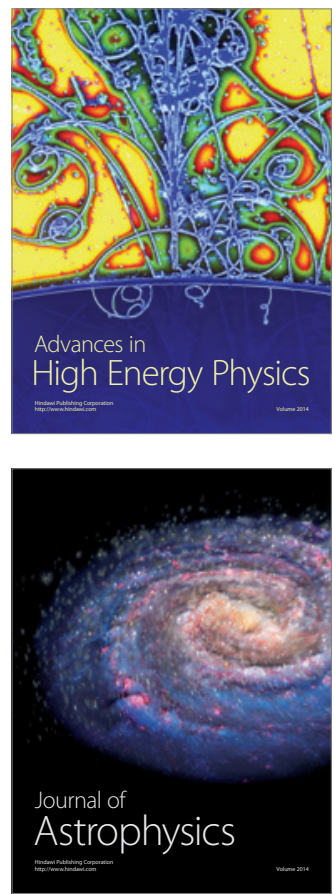
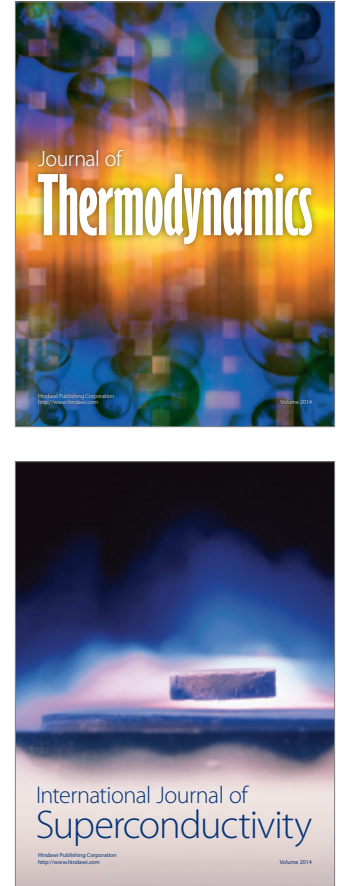
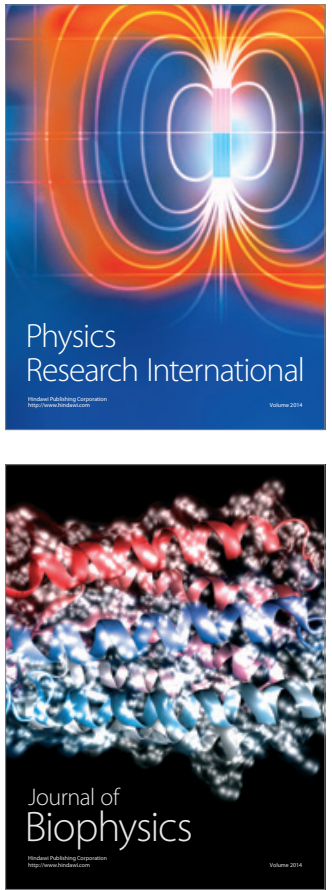
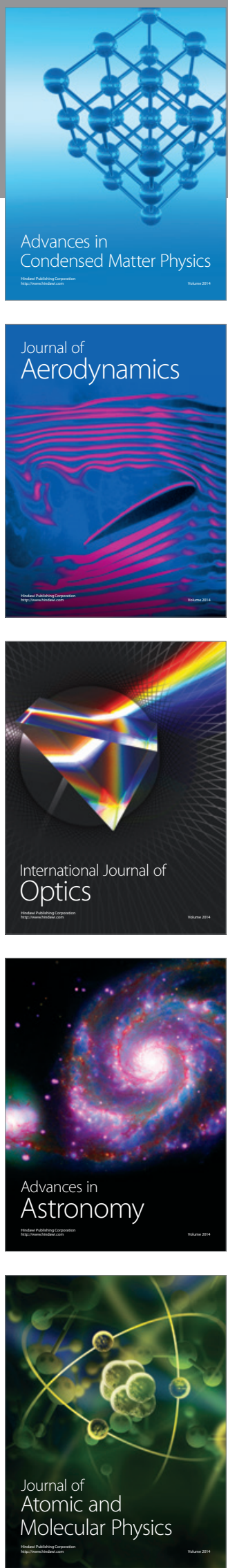